\title{
Avaliação de material impresso sobre Distúrbios de Sono para ação de educação em saúde pediátrica
}

\author{
Evaluation of printed material on Sleep Disorders for \\ Pediatric Health Education Action
}

\author{
Eleida Pereira Camargo ${ }^{1}$, Luciane Bizari Coin de Carvalho ${ }^{2}$, Lucila \\ Bizari Fernandes do Prado ${ }^{3}$, Gilmar Fernandes do Prado ${ }^{3}$
}

1.Designer, Doutora, Professora das Faculdades Oswaldo Cruz/FAITER e Universidade São Judas Tadeu, pesquisadora setor Neuro-Sono, Departamento de Neurologia da Unifesp, São Paulo-SP, Brasil. 2.Psicóloga, Doutora, Departamento de Neurologia e Neurocirurgia, Unifesp, São Paulo-SP, Brasil. 3.Médico, Doutor, Departamento de Neurologia e Neurocirurgia, Unifesp, São Paulo-SP, Brasil.

\begin{abstract}
Resumo
Introdução. A qualidade do sono influencia diretamente o desenvolvimento físico e emocional das crianças. Muitos materiais impressos têm sido elaborados para ações de educação em saúde, mas temos poucos dados estatisticamente sistematizados sobre a eficácia de Histórias em Quadrinhos junto a crianças. Objetivo. Avaliar a utilização da História em Quadrinhos (HQ) "Ronco Dorme em Casa" na ação de educação em saúde pediátrica testando sua eficiência junto a crianças de 6 a 10 anos de idade. Método. 548 crianças de 6 a 10 anos, matriculadas no ensino fundamental de escolas paulistanas das redes pública e privada responderam a 3 questões sobre Distúrbios de sono (teste). Segundo o modelo "Salomon 3 grupos", os estudantes foram divididos em 3 grupos: Grupo Pós-HQ (que respondeu ao teste somente após a leitura da História em Quadrinhos) e Grupo Pré \& Pós-HQ (que respondeu ao teste antes e depois da leitura da História em Quadrinhos). Resultados. As crianças apresentaram mais respostas corretas após a leitura da HQ comparadas as respostas antes da leitura $(p<0,05$ no geral; Questão 1: $p<0,000001$; Questão 2: $p<0,000001$ e Questão 3: $p<0,000001$ ). Nas respostas assinaladas após a leitura da HQ não houve diferença entre procedência escolar, sexo e idade, com exceção da questão 3 , de maior complexidade, onde as crianças mais velhas (10 anos) obtiveram melhor desempenho. Conclusão. Os resultados apontaram a eficácia da HQ "Ronco Dorme em Casa" como coadjuvante na ação de educação em saúde pediátrica.
\end{abstract}

Unitermos. Educação; Distúrbio do Sono; História em Quadrinhos; Design da informação

\begin{abstract}
Introduction. Sleep quality directly influences children's physical and emotional development. Many printed materials have been developed for health education actions, but we have little statistically systematized data on the efficacy of comics with children. Objective. To evaluate the use of comic strips "Snoring lives at Home" in the pediatric health education action testing its efficiency among children from 6 to 10 years of age. Method. 548 children aged 6 to 10 enrolled in elementary school in São Paulo public and private schools answered 3 questions about Sleep Disorders (test). According to the model "Salomon 3 groups", the students were divided into 3 groups: Post-HQ Group (who responded to the test only after reading Comics) and Pre \& Post-HQ Group (who answered the before and after test of the comic book reading). Results. The children presented more correct answers after reading the comic compared to the answers before reading $(p<0.05$ in general; Question 1: $p<0.000001$; Question 2: $p<0.000001$, and Question 3: $p<0,000001)$. In the responses indicated after reading the comic, there was no difference between school origin, sex and age, except for question 3, of greater complexity, where older children (10 years) had better performance. Conclusion. The results pointed to the efficacy of the comic "Snoring lives at Home" as an adjunct to the pediatric health education action.
\end{abstract}

Keywords. Education; Sleep Disorder; Comic; Information Design 
Trabalho realizado na Escola Paulista de Medicina (EPM) da Universidade Federal de São Paulo (Unifesp), São Paulo-SP, Brasil.

\section{INTRODUÇÃO}

Embora exista uma reconhecida necessidade de desenvolvimento e produção de materiais impressos que auxiliem na comunicação entre o profissional de saúde e o paciente, instruindo-o sobre procedimentos, sintomas e tratamentos ${ }^{1}$, pouco tem se avaliado a eficiência da comunicação visual impressa, especificamente voltada a este segmento ${ }^{2}$.

Há uma profusão de materiais impressos associados à divulgação ou promoção de saúde. Já foram catalogados cerca de 250 títulos produzidos para uma única instituição, mas recorrentemente esta produção é realizada sem que haja uma interação entre os envolvidos: profissionais de saúde, designers e pacientes.

Na ação de promoção e comunicação em saúde há o predomínio da visão do especialista em detrimento das expectativas da sociedade e público ao qual esta ação se destina. Não havendo esta preocupação, há poucas informações sobre o processo de desenvolvimento e formulação destes materiais e sua eficácia frente a seus objetivos $^{3}$. O material paradidático avaliado no presente projeto tem por finalidade informar e conscientizar o paciente pediátrico sobre patologias do Sono. 
Quando nos referimos a um distúrbio de sono manifestado em uma idade precoce, podemos dizer que este transtorno traz consequências para toda a família ${ }^{4}$. Crianças que manifestam algumas patologias do sono solicitam sistematicamente a intervenção de um adulto. Não podemos deixar de lembrar também, as implicações de ordem cognitiva. A qualidade do sono interfere no desempenho escolar da criança que pode apresentar: irritabilidade, dificuldades de concentração e memorização. O diagnóstico precoce amplia as possibilidades de tratamentos menos intrusivos e mais eficazes ${ }^{5-7}$.

A Superintendência de Controle de Endemias da Secretaria de Saúde do Estado de São Paulo - SUCEN define Ação Educativa como intervenção que se utiliza das teorias de ensino aprendizagem e comunicação para a difusão de conhecimento e informações nas áreas de ciências biológicas e saúde ${ }^{8}$.

Este projeto utiliza-se do recurso da História em Quadrinhos (HQ) para a difusão deste conhecimento, dirigido a crianças pertencentes a faixa etária de 6 a 10 anos de idade ${ }^{9,10}$. O objetivo do estudo é avaliar a eficácia da HQ "Ronco Dorme em Casa", especialmente desenvolvida para o projeto, nas informações sobre distúrbios de sono, junto ao público infantil. 


\section{MÉTODO}

\section{População e local}

O estudo foi desenvolvido na cidade de São Paulo que conta com cerca de 11 milhões de habitantes concentrados em uma área de $1.509 \mathrm{~km}^{2}$. Trata-se do quinto maior conglomerado urbano do mundo, depois de Tóquio, Cidade do México, Seul e Bombaim ${ }^{11}$.

$\mathrm{Na}$ cidade destacam-se cinco grandes regiões geográficas (norte, sul, leste, oeste e centro) administrativamente divididas em 31 Subprefeituras e 96 Distritos Municipais.

Em 2000, 95,41\% da população residente no município, acima dos 10 anos de idade, era alfabetizada ${ }^{12}$. As maiores taxas de alfabetização eram registradas nas regiões centrais $(99,45 \%)$ e as menores encontravam-se nos distritos periféricos $(89,22 \%)$.

O modelo de escolarização vigente no país constitui-se em três grandes ciclos: educação infantil (2 a 5 anos); ensino fundamental ( 6 a 14 anos) e ensino médio (15 a 17 anos). São Paulo contava com 1.613.435 habitantes matriculadas no ensino fundamental (2006). Na faixa etária de 6 a 10 anos, população de interesse para este estudo, tínhamos 820.537 crianças distribuídas nas redes estadual (375.633 ou 45,78\%), municipal (304.337ou 37,09\%) e particular (140.373 ou $17,11 \%)$. 


\section{Amostra}

O tamanho calculado da amostra foi de 450 indivíduos, para erro amostral de $5 \%$ e intervalo de confiança IC $=95 \%$. Com a finalidade de garantir o mínimo de inclusões previstas por este cálculo, o estudo foi iniciado com 619 crianças, de 6 a 10 anos de idade, no período de $20 / 08$ a 10/09/2008. A amostragem levou em conta as regiões centrais e periféricas da cidade, bem como suas três redes de ensino: pública estadual (291 alunos), pública municipal (179 alunos) e privada (149 alunos).

Excluímos 71 crianças por não apresentarem completa alfabetização ou não pertencerem a faixa etária desta pesquisa. A amostra final contou com 548 crianças, superando o total definido inicialmente para o estudo.

Estes alunos estavam matriculados no ensino fundamental, (de $1^{\text {a }}$ a $4^{\mathrm{a}}$ ano), distribuídos por idade, da seguinte maneira: 204 tinham 10 anos; 199 tenham 9 anos; 102 contavam com 8 anos e 36 alunos encontravamse na faixa etária de 7 a 6 anos.

A pesquisa foi aprovada pelo Comitê de Ética em Pesquisa da Universidade Federal de São Paulo (UNIFESP) sob o protocolo de número 0850/05. Todos os responsáveis pelas crianças assinaram o termo de consentimento.

\section{Randomização e formação dos Grupos}

Foi utilizado o modelo "Salomon 3 grupos" indicado para estudos em que deve haver uma medida anterior a intervenção ${ }^{13,14}$. Este procedimento metodológico 
caracteriza-se pela existência de dois grupos teste: um deles somente com avaliação posterior a intervenção (Grupo Pós-HQ) e outro com avaliações anterior e posterior a intervenção (Grupo Pré \& Pós-HQ). O grupo que é avaliado apenas posteriormente evita que a atenção sobre o assunto seja despertada por meio das questões propostas em pré-teste, minimizando influências sobre os resultados no pós-teste.

Os grupos foram definidos por sorteio aleatório das 26 turmas participantes. O número máximo de salas em cada escola foi 15 e o mínimo 8, distribuídas nos períodos matutino e vespertino. No processo de sorteio, cada sala a ser incluída recebeu um número consecutivo que foi registrado em um cartão. Todos os cartões numerados foram colocados em uma caixa e agitados de modo a ficarem completamente embaralhados. Após este procedimento retirávamos $50 \%$ dos cartões com a finalidade de formar o grupo Pós-HQ, sendo os cartões restantes na caixa os que constituíram o grupo Pré \& Pós$\mathrm{HQ}$. Tivemos uma diferença do número de crianças incluídas em cada grupo, porque 0 método de randomização levou em conta as salas pré-existentes nas escolas. No grupo Pós-HQ, houve ainda a perda de duas turmas, cujos alunos encontravam-se em atividade externa, no dia em que foi realizada a pesquisa.

O grupo Pós-HQ foi constituído de 232 crianças que foram avaliadas apenas após a leitura da HQ (intervenção), enquanto o grupo Pré \& Pós-HQ foi formado por 316 
crianças que responderam às mesmas questões antes e depois da leitura da HQ.

\section{História em Quadrinhos}

A HQ desenvolvida para este estudo, "Ronco dorme em casa"15, aborda dois dos temas de distúrbios do sono: Higiene do Sono e a Síndrome do Ronco. Conta uma história sobre duas crianças que se preparam para adormecer. Neste contexto são apresentadas algumas recomendações como: não comer na cama, não praticar exercícios físicos imediatamente antes de dormir e fazer alimentação leve. O ronco também é citado, apresentandose como anormal, apesar de sua popular banalização, alertando para o fato de que pode ser um sintoma de um distúrbio respiratório importante.

\section{Instrumento de verificação de conhecimento}

$O$ instrumento utilizado para a avaliação antes e após a leitura da $\mathrm{HQ}$ foi um teste (Figura 1) constituído de três perguntas fechadas sobre higiene do sono e a síndrome do ronco.

O nível de complexidade das questões foi crescente. A primeira pergunta foi objetiva, com três possibilidades de respostas baseadas em ações rotineiras ilustradas por imagens com o propósito de verificar conhecimentos em um plano concreto de entendimento, além da noção primitiva de bom ou mau, sem exigir conceitos de saúde ou doença. A segunda questão pressupõe um conceito prévio 
de normalidade e saúde com a resposta dicotomizada em sim ou não. $\mathrm{Na}$ terceira questão solicitou-se 0 estabelecimento de relação causa e efeito em três assertivas: a primeira levava em conta a percepção da criança de que o ronco é algo comum e, portanto, inerente as pessoas; a segunda apresentava o ronco como um sintoma e a terceira levava em conta o comportamento socialmente adequado.

Figura 1. Instrumento de avaliação (Teste respondido pelas crianças, antes e depois da leitura da HQ)

Idade $\operatorname{sexo}() \mathrm{F} \quad(\quad) \mathrm{M}$

1. MARQUE COM UM "X" O QUE É BOM FAZER ANTES DE DORMIR.

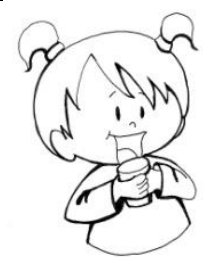

a) TOMAR LEITE

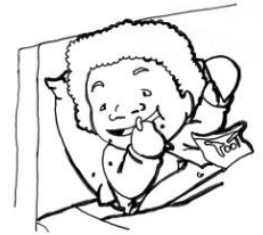

c) COMER NA CAMA

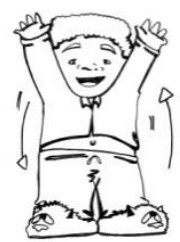

d) FAZER EXERCÍCIOS

2. RONCAR É NORMAL? ( ) SIM （）NÃO .

ABAIXO, MARQUE COM UM "X" PORQUE VOCÊ ACHA ISSO.

a) PORQUE TODO MUNDO RONCA.

b) PORQUE É UM SINAL DE QUE ALGO ESTÁ ERRADO.

c) PORQUE NÃO É EDUCADO. 
Sua elaboração considerou o nível de alfabetização das crianças pertencentes à amostra, utilizando-se de vocabulário acessível, textos breves e imagens ${ }^{26-28}$. Tanto a $\mathrm{HQ}$ quanto o instrumento de verificação foram previamente testados em pesquisa piloto ${ }^{15}$, e se mostraram adequados a compreensão da faixa etária pretendida (6 a 10 anos $)^{16}$.

\section{Intervenção}

O questionário foi aplicado diretamente pela pesquisadora em 26 salas de aula, com a presença das respectivas professoras. As crianças foram apresentadas à pesquisadora que em seguida as informou sobre o estudo dizendo apenas que se tratava da leitura de uma história em quadrinhos não relacionada ao conteúdo acadêmico. Portanto a atividade seria anônima e não Ihes seria atribuída nota, uma vez que a $\mathrm{HQ}$ era o objeto a ser avaliado e não as crianças.

Nas salas do grupo Pós-HQ, a HQ foi imediatamente entregue aos alunos, após as instruções descritas acima. Assim que as crianças concluíram a leitura da história, receberam 0 instrumento de verificação (teste) e o preencheram sem auxílio.

Nas salas do grupo Pré \& Pós-HQ, as mesmas instruções foram dadas, porém as crianças responderam ao teste também antes da intervenção. Inicialmente receberam $o$ instrumento de verificação e somente após o responderem, iniciaram a leitura da $\mathrm{HQ}$. Ao finalizarem a leitura, as crianças responderam novamente ao teste 
aplicado anteriormente, com a finalidade de se avaliar o conhecimento adquirido após a intervenção da HQ. O tempo médio para a realização dos procedimentos foi de 15 minutos em cada uma das salas estudadas.

\section{Desfechos e análise dos dados}

O desfecho esperado para este estudo foi a assimilação dos conceitos relacionados higiene do sono e síndrome de ronco. Após a leitura da HQ (intervenção) esperava-se que as crianças, identificassem que seriam hábitos saudáveis: tomar leite antes de dormir, não comer na cama e não se exercitar imediatamente antes do sono. Quanto ao ronco, esperava-se que as crianças o identificassem como um sintoma.

Os temas considerados para as análises foram a higiene do sono e o ronco. A higiene do sono subdividiu-se em três variáveis: "tomar leite", "comer na cama" e "fazer exercícios". O ronco subdividiu-se em cinco variáveis: na questão 2, em: "normal" e "anormal" e na questão 3 em: "todo mundo ronca"; "é sinal de que algo está errado" e "não é educado". A leitura da HQ, entendida como uma intervenção foi avaliada em relação às oito variáveis citadas. Calculamos para cada variável os riscos absolutos, relativos e o NNT. Entendemos o NNT, à semelhança do modelo utilizado em intervenções com medicamentos, como o número necessário para mudança conceitual, ou seja, o número mínimo de crianças necessárias para que o resultado desejado ocorresse. 


\section{Análise estatística}

Analisamos os dados através de estatística descritiva e os mesmos serão apresentados como média e desvio padrão (média \pm desvio padrão). Com a finalidade de verificarmos o impacto da $\mathrm{HQ}$ na assimilação dos conceitos sobre higiene do sono e ronco, calculamos o risco absoluto, risco relativo, IC 95\% e o número necessário para tratar (NNT) nos grupos Pós-HQ e Pré \& Pós-HQ. Utilizamos o teste do Qui-quadrado para analisar as respostas assinaladas entre os grupos e intragrupo, bem como para os grupos formados a partir das variáveis: faixa etária, gênero e escola, constituídos de forma dicotômica. Foi considerado significante $p<0,05$.

\section{RESULTADOS}

\section{Dados Demográficos}

Os grupos Pós-HQ e Pré \& Pós-HQ não apresentaram diferenças quanto à idade, gênero e procedência escolar (pública municipal, pública estadual e privada). O grupo Pós-HQ compôs-se de 232 crianças e o grupo Pré \& Pós$\mathrm{HQ}$, de 316 crianças. A média etária da amostra total foi de $9,3 \pm 1,2$ anos. A média de idade no grupo Pós-HQ foi de

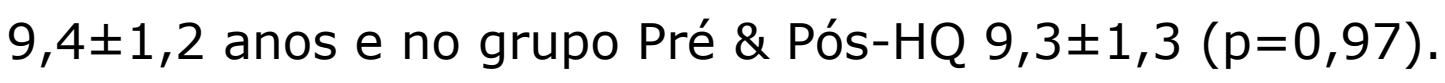

Quanto à variável gênero, os grupos também se mostraram homogêneos. No grupo Pós-HQ, 43,96\% $(\mathrm{N}=102)$ eram do sexo masculino e $56,03 \%(\mathrm{~N}=130)$ do feminino; e no grupo Pré \& Pós-HQ, 42,09\% ( $N=133)$ eram 
do sexo masculino e $57,91 \%$ (183) do sexo feminino $(p=0,705)$.

No grupo Pós-HQ 71,12\% eram de escolas públicas e $27,59 \%$ eram de escolas privadas; e no grupo Pré \& PósHQ $75,62 \%$ era oriunda de escolas públicas e $24,37 \%$ de escolas particulares $(p=0,46)$.

\section{Higiene do sono}

Grupo Pré \& Pós-HQ. Antes da leitura da HQ, dentre as 316 crianças deste grupo, 66,8\% assinalou que "tomar leite" antes de dormir é um bom hábito; 10,4\% considerou que "comer na cama" é correto e 38,0\% apontou que "fazer exercícios" seria indicado. Após a leitura da HQ, o percentual de crianças que assinalou as opções "tomar leite", "comer na cama" e "fazer exercícios" foi respectivamente: $84,5 \%$, diferença de $17,7 \%, p<0,0001$; $4,4 \%$, diferença de $6 \%, p<0,0005 ; 15,5 \%$, diferença de 22,5\%, p<0,00001 (Tabela 1, Figura 2).

Tabela 1. Comparação Grupo Pré e Pós-HQ para as 316 crianças.

\begin{tabular}{|c|c|c|c|c|c|c|}
\hline \multirow[b]{2}{*}{ Higiene do sono } & \multicolumn{6}{|c|}{ O que é bom fazer antes de dormir? } \\
\hline & \multicolumn{2}{|c|}{ Tomar leite \% $(\mathrm{N})$} & \multicolumn{2}{|c|}{$\begin{array}{c}\text { Comer na cama \% } \\
(\mathrm{N})\end{array}$} & \multicolumn{2}{|c|}{ Fazer exercícios \% (N) } \\
\hline \multirow{2}{*}{$\begin{array}{l}\text { INTRAGRUPO } \\
\text { P-PHQ } \\
\text { Pré \& Pós-HQ }\end{array}$} & $\begin{array}{l}\text { Antes } \\
\mathbf{6 6 , 8 \%} \\
(\mathbf{2 1 1})\end{array}$ & $\begin{array}{l}\text { Depois } \\
\mathbf{8 4 , 5 \%} \\
(\mathbf{2 6 7 )}\end{array}$ & $\begin{array}{c}\text { Antes } \\
10,4 \% \\
(33)\end{array}$ & $\begin{array}{c}\text { Depois } \\
4,4 \% \\
(14)\end{array}$ & $\begin{array}{l}\text { Antes } \\
38 \% \\
(120)\end{array}$ & $\begin{array}{c}\text { Depois } \\
15,5 \% \\
(49)\end{array}$ \\
\hline & \multicolumn{2}{|c|}{$\begin{array}{c}\Delta 17,7 \%(56) \\
\mathrm{p}<0,00001\end{array}$} & \multicolumn{2}{|c|}{$\begin{array}{c}\Delta-6 \%(-19) \\
\mathrm{p}<0,0005\end{array}$} & \multicolumn{2}{|c|}{$\begin{array}{c}\Delta-22,5 \%(-71) \\
\mathrm{p}<0,00001\end{array}$} \\
\hline \multirow{2}{*}{$\begin{array}{c}\text { INTERGRUPO } \\
\text { P-PHQ X PHQ } \\
\text { Pós } \\
\text { Intervenção }\end{array}$} & $\begin{array}{c}\text { P-PHQ } \\
84,5 \% \\
(267) \\
\end{array}$ & $\begin{array}{c}\text { PHQ } \\
86,2 \% \\
(200) \\
\end{array}$ & $\begin{array}{c}\text { P-PHQ } \\
4,4 \% \\
(14) \\
\end{array}$ & $\begin{array}{c}\mathrm{PHQ} \\
4,7 \% \\
(11) \\
\end{array}$ & $\begin{array}{c}\mathrm{P}-\mathrm{PHQ} \\
15,5 \% \\
(49) \\
\end{array}$ & $\begin{array}{c}\mathrm{PHQ} \\
11,2 \% \\
(26)\end{array}$ \\
\hline & \multicolumn{2}{|c|}{$\Delta 1,7 \%$} & \multicolumn{2}{|c|}{$\Delta 0,3 \%$} & \multicolumn{2}{|c|}{$\Delta 4,3 \%$} \\
\hline
\end{tabular}


Figura 2. Percentual de crianças do grupo Pré \& Pós-HQ que responderam aos três itens da questão 1 , pré e pós leitura da HQ.

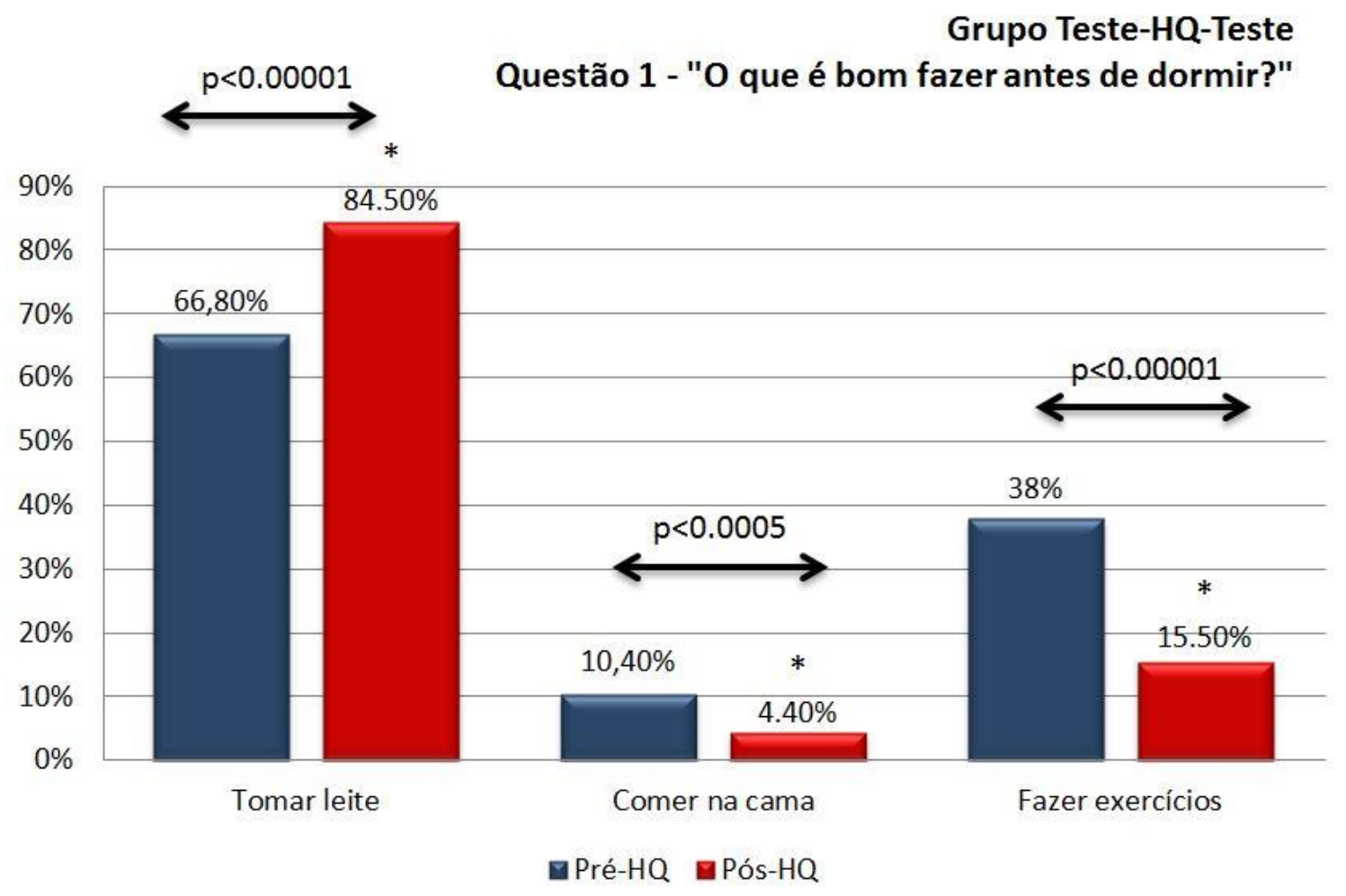

Sexo, Idade e Escola (Pré \& Pós-HQ). Antes da intervenção, os meninos assinalaram $12,3 \%$ mais vezes a opção "fazer exercícios" do que as meninas $(p=0,02)$. Observamos 93,51\% de acertos na escola particular, 84\% na municipal e $80,49 \%$ na estadual, ou seja, as crianças provenientes do estabelecimento particular demonstraram maior conhecimento prévio sobre os benefícios da ingestão do leite, antes de dormir $(p=0,000005)$. Após a intervenção, não houve diferença entre o número de acertos para as variáveis sexo, idade e procedência escolar (respectivamente, $p=0,49713 ; p=0,86534$ e $p=0,07867$; Tabela 1, Figura 3). 
Figura 3. Percentual de crianças do grupo Pré \& Pós-HQ que respondeu corretamente à questão "o que é bom fazer antes de dormir", em pré-teste, segundo a escola de origem.

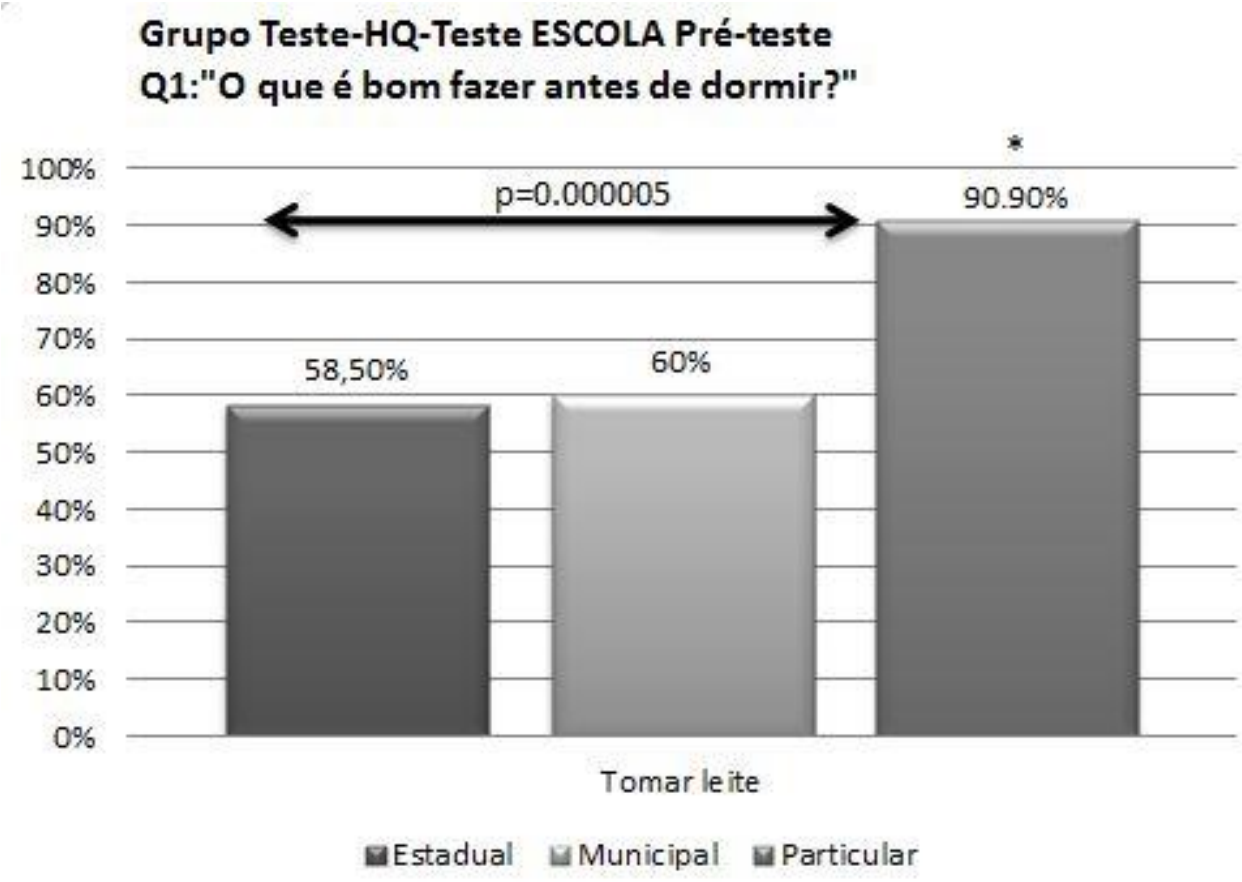

Grupo Pós-HQ. Neste grupo, das 232 crianças, 86,2\% assinalou que "tomar leite" é indicado antes de dormir; $4,7 \%$ apontaram que "comer na cama" é correto e 11,2\% assinalaram que "fazer exercícios" também é um hábito saudável (Tabela 1 ).

Impacto do Pré-teste na pós-intervenção (Pré \& Pós$H Q \times$ Pós-HQ). Responder as questões antes da leitura da HQ não promoveu diferenças no teste após a HQ para duas variáveis que abordaram higiene do sono ("tomar leite, $p=0,4$; "comer na cama", $p=0,8)$. "Fazer exercícios" foi considerado correto por $15,5 \%$ das crianças do grupo Pré \& Pós-HQ e por $11,2 \%$ das do grupo Pós-HQ ( $p=0,02$; Tabela 1). 


\section{Ronco}

Grupo Pré \& Pós-HQ. Antes da leitura da HQ, 35,4\% das crianças assinalou que roncar é anormal e 57,9\% que é normal. Após a leitura da HQ 51,3\% considerou que roncar é anormal (diferença de 15,8\%; $p<0,00001$ ) e 37,3\% que roncar é normal (diferença de 20,6\%; p<0,00001; Tabela 2 Figura 4).

Sexo, Idade e Escola (Pré \& Pós-HQ). Antes e após a leitura da $H Q$, não houve diferença estatisticamente significante entre sexo, idade e procedência escolar na proporção de respostas corretas ou incorretas.

Grupo Pós-HQ. Para 43,1\% das crianças roncar é anormal e para 50,0\% é normal (Tabela 2).

Impacto do Pré-teste na pós-intervenção (Pré \& Pós$H Q$ x Pós-HQ). Responder as questões antes da intervenção promoveu diferenças entre os grupos. Roncar foi anormal para $43,1 \%$ das crianças do grupo Pós-HQ e 51,3\% do grupo Pré \& Pós-HQ $(p=0,003)$.

Tabela 2. Grupo Pré \& Pós-HQ. Antes da leitura da HQ, para a questão: roncar é normal.

\begin{tabular}{|c|c|c|c|c|}
\hline \multirow[t]{2}{*}{ Ronco } & \multicolumn{4}{|c|}{ Roncar é normal? } \\
\hline & \multicolumn{2}{|c|}{$\operatorname{Sim} \%(N)$} & \multicolumn{2}{|c|}{ Não \% (N) } \\
\hline \multirow{4}{*}{$\begin{array}{c}\text { INTRAGRUPO } \\
\text { P-PHQ Pré \& } \\
\text { Pós-HQ }\end{array}$} & Antes & Depois & Antes & Depois \\
\hline & $\begin{array}{c}57,9 \% \\
(183)\end{array}$ & $37,3 \%(118)$ & $\begin{array}{c}35,4 \% \\
(112)\end{array}$ & $\begin{array}{c}51,3 \% \\
(162)\end{array}$ \\
\hline & \multirow{2}{*}{\multicolumn{2}{|c|}{$\Delta-20,6 \%(-65)$}} & \multicolumn{2}{|c|}{$\Delta 15,8 \%(50)$} \\
\hline & & & $\mathrm{p}<$ & 001 \\
\hline \multirow{4}{*}{$\begin{array}{l}\text { INTERGRUPO } \\
\text { P-PHQ X PHQ } \\
\text { Pós } \\
\text { Intervenção }\end{array}$} & P-PHQ & PHQ & P-PHQ & PHQ \\
\hline & $37,3 \%$ & $50,0 \%(116)$ & $51,3 \%$ & $43,1 \%$ \\
\hline & $\Delta 12,7 \%$ & $7 \%$ & $(162)$ & $\frac{(100)}{10}$ \\
\hline & \multicolumn{2}{|c|}{$p=0,0034$} & \multicolumn{2}{|c|}{$p<0,00001$} \\
\hline
\end{tabular}


Figura 4. Percentual de crianças do grupo Pré \& Pós-HQ que responderam à questão 2, pré e pós leitura da HQ.

\section{Grupo Teste-HQ-Teste Questão 2 - "Roncar é normal?"}

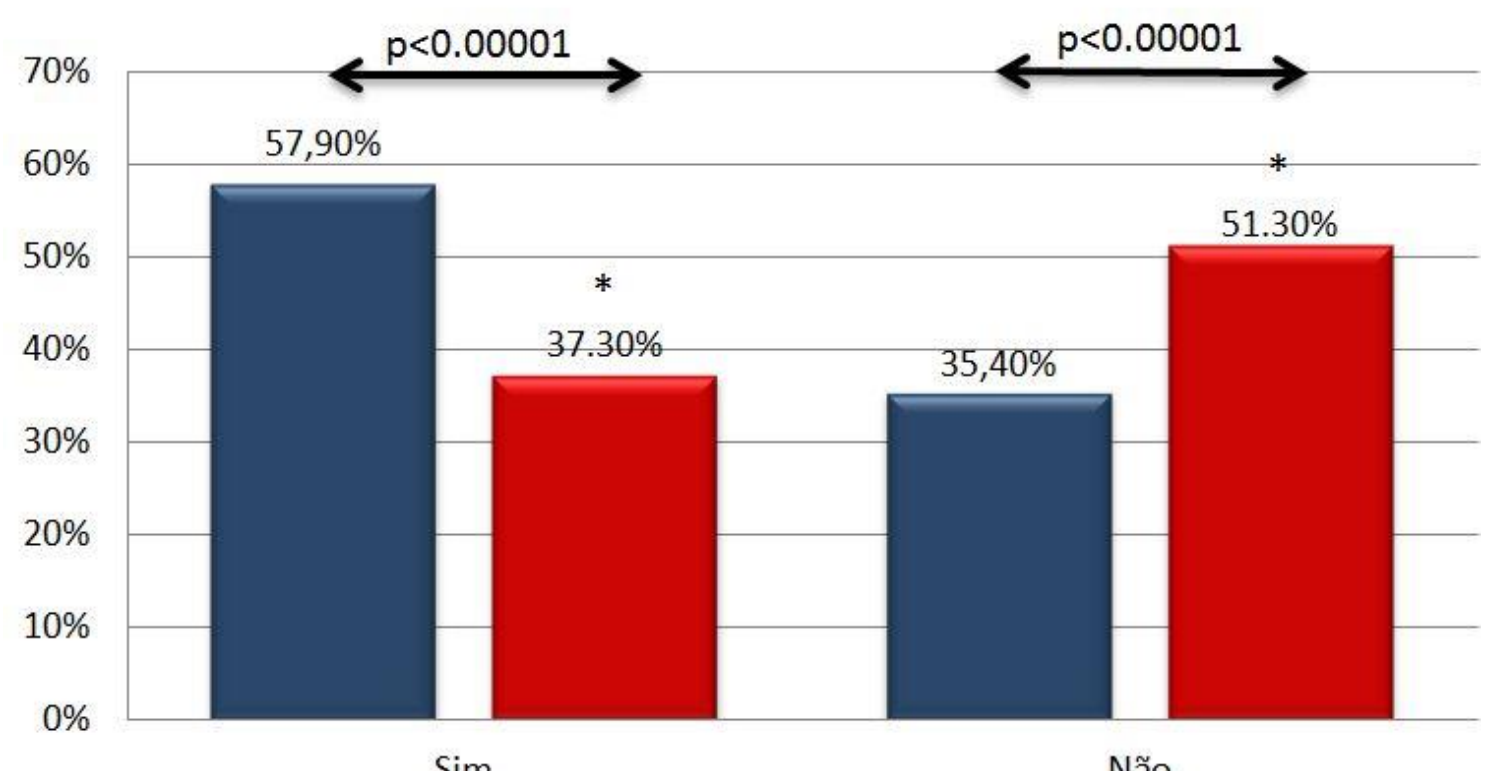

Sim

Não

口Pré-HQ घPós-HQ

\section{Significado do ronco}

Grupo Pré \& Pós-HQ. Antes da leitura da HQ, 39,6\% das crianças identificou o ronco como um possível sintoma, assinalando que é indicativo de que "algo está errado"; $42,1 \%$ considerou que "todo mundo ronca" e $23,4 \%$ assinalou que roncar "não é educado". Após a leitura da HQ, observamos que $61,4 \%$ das crianças passou a identificar o ronco como sintoma (aumento 21,8\%; p<0,00001, Tabela 3); 25,6\% assinalou que "todo mundo ronca" e 19,0\% que "não é educado" (diminuindo, respectivamente $16,5 \% ; p<0,00001$ e 4,4\%; $p=0,06$; Figura 5). 
Tabela 3. Grupo Pré \& Pós-HQ. Indicando o motivo do ronco.

\begin{tabular}{|c|c|c|c|c|c|c|}
\hline Ronco & \multicolumn{6}{|c|}{ Porque você acha isso? } \\
\hline \multirow{4}{*}{$\begin{array}{c}\text { INTRAGRUPO } \\
\text { P-PHQ Pré \& } \\
\text { Pós-HQ }\end{array}$} & \multicolumn{2}{|c|}{ Todo mundo ronca $\%(\mathrm{~N})$} & \multicolumn{2}{|c|}{$\begin{array}{c}\text { É sinal de que algo está } \\
\text { errado } \%(N)\end{array}$} & \multicolumn{2}{|c|}{ Não é educado \% (N) } \\
\hline & $\begin{array}{c}\text { Antes } \\
42,1 \%(133)\end{array}$ & $\begin{array}{c}\text { Depois } \\
25,6 \% \\
(81)\end{array}$ & $\begin{array}{c}\text { Antes } \\
\mathbf{3 9 , 6 \%} \\
(\mathbf{1 2 5})\end{array}$ & $\begin{array}{l}\text { Depois } \\
\mathbf{6 1 , 4 \%} \\
(\mathbf{1 9 4 )}\end{array}$ & $\begin{array}{c}\text { Antes } \\
23,4 \%(74)\end{array}$ & $\begin{array}{c}\text { Depois } \\
19,0 \%(60)\end{array}$ \\
\hline & \multicolumn{2}{|c|}{$\Delta-52(-16,5 \%)$} & \multicolumn{2}{|c|}{$\Delta 21,8 \%(19)$} & \multicolumn{2}{|c|}{$\Delta-4,4 \%(-71)$} \\
\hline & \multicolumn{2}{|c|}{$\mathrm{p}<0,00001$} & \multicolumn{2}{|c|}{$\mathrm{p}<0,00001$} & \multicolumn{2}{|c|}{$P=0,06$} \\
\hline \multirow{2}{*}{$\begin{array}{l}\text { INTERGRUPO } \\
\text { P-PHQ X PHQ } \\
\text { Pós } \\
\text { Intervenção }\end{array}$} & $\begin{array}{c}\text { P-PHQ } \\
25,6 \%(81)\end{array}$ & $\begin{array}{c}\mathrm{PHQ} \\
39,2 \% \\
(91)\end{array}$ & $\begin{array}{l}\text { P-PHQ } \\
\mathbf{6 1 , 4 \%} \\
(\mathbf{1 9 4 )}\end{array}$ & $\begin{array}{c}\mathrm{PHQ} \\
\mathbf{4 9 , 6 \%} \\
(\mathbf{1 1 5})\end{array}$ & $\begin{array}{c}\text { P-PHQ } \\
19,0 \%(60)\end{array}$ & $\begin{array}{c}\mathrm{PHQ} \\
18,1 \% \\
(42)\end{array}$ \\
\hline & \multicolumn{2}{|c|}{$\begin{array}{c}\Delta 13,6 \% \\
p<0,00001\end{array}$} & \multicolumn{2}{|c|}{$\begin{array}{c}\Delta 11,8 \% \\
p=0,00003\end{array}$} & $\begin{array}{l}\Delta 0 \\
\mathrm{P}=1\end{array}$ & $9 \%$ \\
\hline
\end{tabular}

Figura 5. Percentual de crianças do grupo Pré \& Pós-HQ que responderam aos 3 itens da questão 3, pré e pós leitura da HQ.

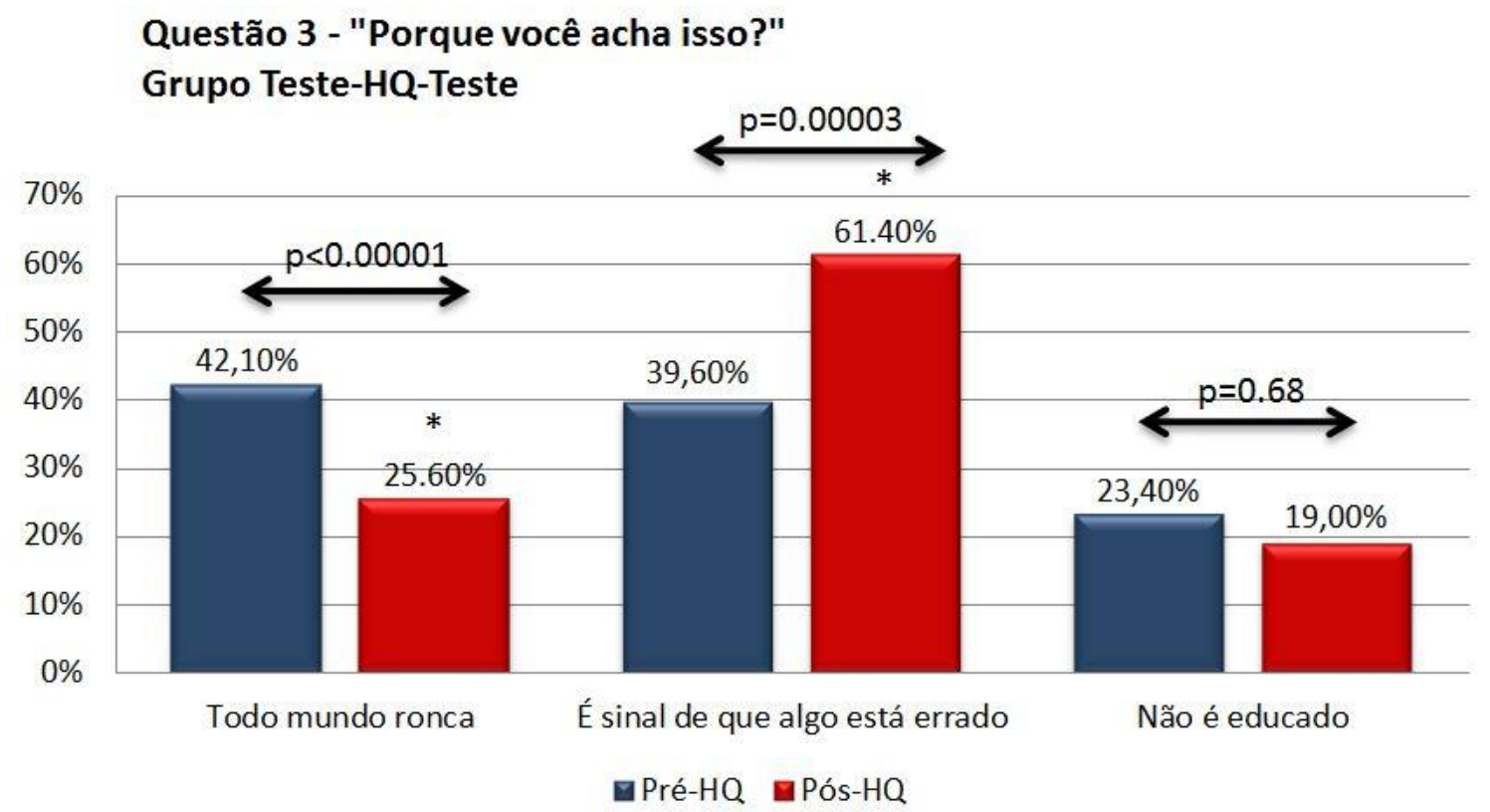

Sexo, Idade e Escola (Pré \& Pós-HQ). Não se observou diferenças estatisticamente significantes na proporção de respostas corretas quando levamos em conta o sexo e procedência escolar. Após a leitura da $\mathrm{HQ}, 72,4 \%$ das crianças mais velhas (10 anos) assinalaram que o ronco 
indica algum problema de saúde ( $p=0,005 ;$ Tabela 3$) .0$ aumento de acertos correlaciona-se positivamente com 0 aumento da idade, $(r=0,97 ; p=0,005$; Tabela 3; Figura 6$)$.

Figura 6. Percentual de crianças do grupo Pré \& Pós-HQ, que respondeu corretamente à questão "Porque você acha isso?" (indicando o ronco como possível sinal de doença), no pós-teste, segundo faixa etária.

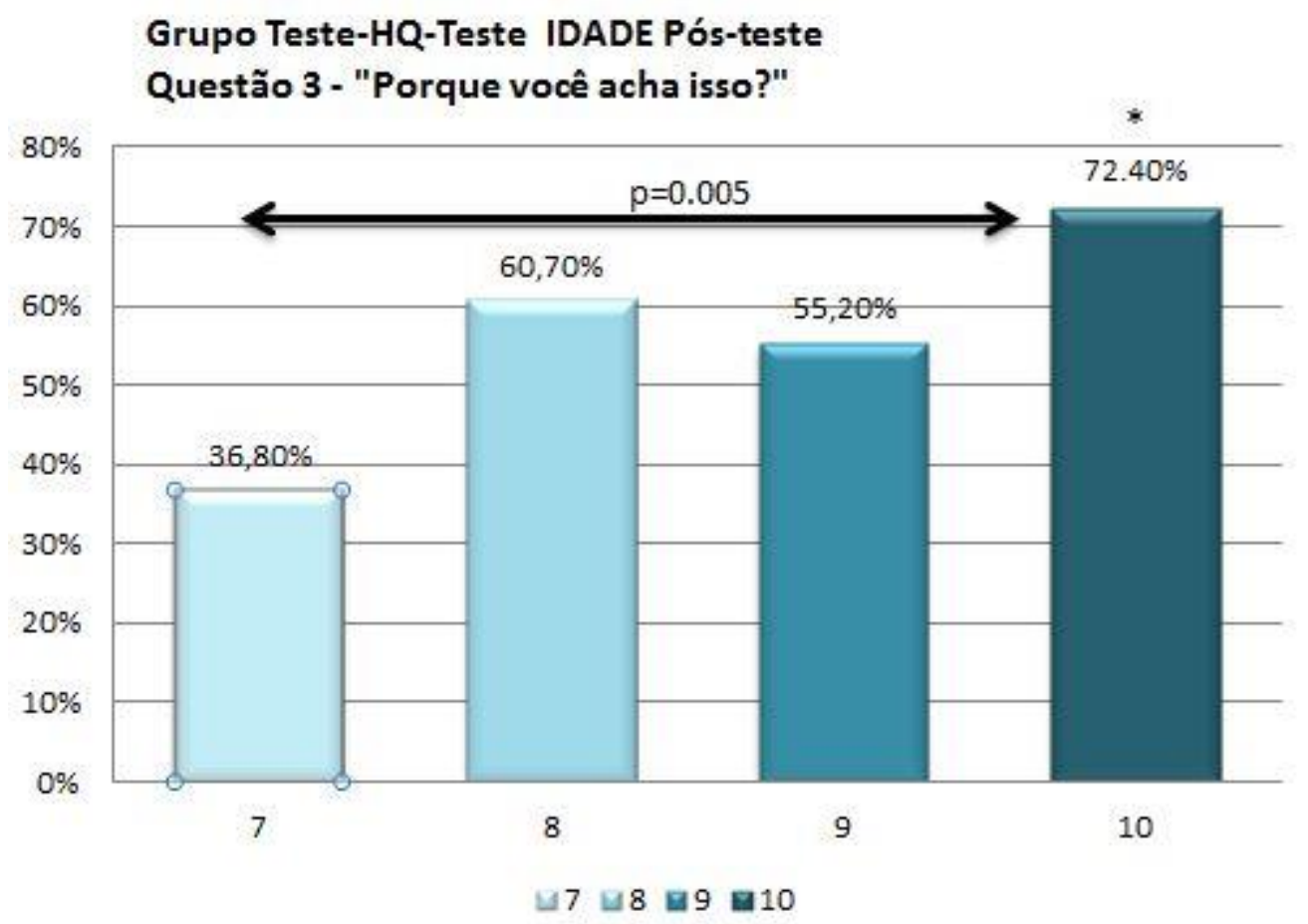

Grupo Pós-HQ. Neste grupo 49,6\% dos estudantes identificou o ronco como sintoma, assinalando que "algo está errado"; 39,2\% considerou que "todo mundo ronca" e $18,1 \%$ assinalou que roncar "não é educado".

Impacto do Pré-teste na pós-intervenção (Pré \& Pós$H Q$ x Pós-HQ). A proporção de acertos no grupo Pré \& Pós$\mathrm{HQ}$, para a questão "é sinal de que algo está errado" foi de 61,4\% e no Pós-HQ 49,6\% (diferença de 11,8\%; $p=0,00003)$. A proporção de crianças que assinalou a 
questão "todo mundo ronca" foi $25,6 \%$ no grupo Pré \& PósHQ e 39,2\% no grupo Pós-HQ (diferença de 13,6\%; $p<0,00001)$. Para a assertiva "não é educado" não se observou diferença entre os grupos (Tabela 3 ).

Impacto do Pré-teste no grupo Pré \& Pós-HQ. Neste grupo as crianças foram avaliadas quanto aos conceitos inclusos na $\mathrm{HQ}$ em dois momentos: antes e depois da leitura da história, permitindo se calcular o efeito da intervenção neste grupo através de indicadores clássicos como os riscos absoluto e relativo e o número necessário para tratar (NNT). Ler a HQ associou-se a maior número de acertos sobre os conceitos de higiene do sono $(R R=1,8$; IC 95\% 1,4 a 2,2; p<0,00001; NNT=3,2; Tabela 1; Figura 2), sobre o significado do ronco $(R R=1,4$; IC 95\% 1,2 a 1,6; $\mathrm{p}<0,00001$; NNT=5; Tabela 2; Figura 3) e suas possíveis justificativas ( $R R=1,6$; IC 95\% 1,3 a 1,8; $p<0,00001$; NNT=5; Tabela 3; Figura 5).

\section{DISCUSSÃO}

A HQ "Ronco dorme em casa" foi eficiente em informar sobre higiene do sono e ronco para o público infantil de nosso estudo. Observamos um aumento de respostas corretas e diminuição de respostas incorretas sugerindo que a associação imagem-texto, criada em nossa $H Q$, auxilia a compreensão do conteúdo global, notadamente no caso de indivíduos que se encontraram em processo inicial de alfabetização ${ }^{17}$. 
A utilização da $\mathrm{HQ}$ como recurso didático já foi polêmica no Brasil, chegando a ser vista até como uma ferramenta de colonização e perversão que poderia levar a desvios de conduta e incapacitação intelectual ${ }^{18}$. No entanto é um poderoso instrumento de comunicação do gênero de literatura icônico/verbal que pode se prestar, dependendo de seu conteúdo, a informar e influenciar variadas populações. Os quadrinhos constituem uma das seções mais lidas dos jornais, pois este gênero literário permite o contato com a informação de forma coloquial e lúdica, havendo dados que indicam sua eficiência como recurso didático ${ }^{19,20}$.

Campanhas de Saúde Pública desenvolvidas por organismos mundiais, como a Organização Panamericana de Saúde (OPAS) e a World Health Organization (WHO) $)^{21,22}$, utilizam-se de HQs para esclarecer a população sobre doenças e sua prevenção. Contudo, não há dados sistematizados cientificamente que avaliem isoladamente a eficiência da $\mathrm{HQ}$ na transmissão das informações sobre saúde, pois as campanhas preventivas integram outros recursos como audiovisuais, palestras, exames clínicos, entre outros, cuja efetividade é avaliada conjuntamente $e^{23,24}$.

Pré-HQ. Observarmos em nosso estudo que as crianças questionadas antes da intervenção demonstraram conhecimento prévio em relação à higiene do sono. Mesmo antes de ter recebido informações sobre o tema, as respostas dadas pelos estudantes indicaram que eles 
sabem que "tomar leite" antes de se deitar pode ser benéfico ${ }^{25,26}$, sugerindo ser este um hábito culturalmente estabelecido em nossa população. "Fazer exercícios" antes de dormir foi apontado pelas crianças como uma atividade positiva, principalmente pelos meninos. Acreditamos que esta opção foi assinalada com mais frequência devido a ampla difusão dos benefícios da atividade física tanto no ambiente escolar quanto na mídia ${ }^{27}$. Verificamos ainda que o número de acertos sobre a síndrome do ronco foi inferior aos conceitos relacionados a higiene do sono, provavelmente por versar sobre um conhecimento mais específico, ainda pouco correlacionado a patologias ${ }^{28}$.

Pós-HQ Após a intervenção, observamos diferenças estatisticamente significantes quanto a compreensão global dos temas abordados na $\mathrm{HQ}$, indicando que as crianças reviram seus conceitos a partir desta leitura. A questão objetiva sobre a Higiene do sono obteve maior número de respostas corretas, redução das incorretas e de duplicidades. Contudo, o percentual de abstenções e respostas duplicadas aumentou proporcionalmente em relação a complexidade das questões, indicando indecisões e ideias conflitantes que passam a surgir a partir das novas informações recebidas.

Os resultados foram inferiores quando mensuramos a capacidade das crianças estabelecerem relações de causa e efeito. Cerca de $10 \%$ das crianças consideraram simultaneamente o ronco como manifestação normal e sintoma de uma doença. 
Após a intervenção observamos que as crianças mais velhas tiveram desempenho superior as crianças mais novas à medida que se aumentava a complexidade das proposições sugerindo, como esperado, que as informações contidas na HQ "Ronco dorme em casa" foram absorvidas de forma mais eficiente pelas crianças maiores. O gênero e a escola de origem não tiveram influência nos resultados, sendo um indício de que ao receber a informação contida na $H Q$, ela foi assimilada com relativa homogeneidade, mesmo entre indivíduos com características sociodemográficas distintas.

Comparativamente, também pudemos verificar que o número de acertos foi superior no grupo Pré \& Pós-HQ em relação ao grupo Pós-HQ. Entre os grupos, há sutis diferenças percentuais nas questões sobre higiene do sono, mas alterações expressivas nas perguntas relacionadas a Síndrome do Ronco (questões 2 e 3). A variação de respostas entre os grupos passa a ser estatisticamente mais significante na medida em que aumenta também a complexidade das questões.

Isto pode sinalizar-nos que a abordagem do assunto antes da realização da intervenção pode ter influído na atenção dispensada ao tema e, consequentemente, na leitura do texto proposto, levando a um número maior de acertos após a intervenção. Este é um efeito esperado e mensurável na metodologia aplicada: "Salomon 3 grupos"14. 
É interessante destacar que Roncar foi considerada uma atitude deseducada, pelas crianças, antes e após a leitura da HQ. Este foi o único conceito que não apresentou diferenças estatisticamente significantes entre os dados obtidos em pré e pós intervenção (Pré \& Pós-HQ) e na comparação intergrupos (Pré \& Pós-HQ x Pós- HQ). Tal achado pode ser explicado pelo fato de que a educação é um conceito abrangente relacionado a convenções sociais e comportamentais que não foram abordadas no conteúdo da história "Ronco dorme em casa", portanto, os resultados registraram uma percepção prévia das crianças, sobre a qual não foi feita uma intervenção direta, justificando a ausência de variação de respostas mesmo após a intervenção.

A HQ "Ronco dorme em casa", que aborda os temas higiene do sono e a síndrome do ronco é a primeira de uma série de impressos a serem aplicados em ações de educação em saúde envolvendo o sono. Esta é apenas uma das versões de histórias protagonizadas pelos personagens criados que versam sobre outros distúrbios e informações pertinentes ao tema, determinadas segundo prevalências do atendimento ambulatorial ${ }^{29,30}$.

\section{CONCLUSÃO}

$\mathrm{O}$ estudo demonstrou que a $\mathrm{HQ}$ "Ronco dorme em casa" foi eficaz em informar sobre higiene do sono e ronco, temas abordados textual e iconograficamente na história, 
sugerindo que a $\mathrm{HQ}$ possa ser útil a políticas públicas e a profissionais da saúde e educação envolvidos com crianças nesta faixa etária.

\section{REFERÊNCIAS}

1.Ottawa Charter for Health. Editorial. Health Promotion 1987;1:3-5. 2.Rozemberg B, Silva APP, Vasconcelos-Silva PR. Impressos Hospitalares e a Dinâmica de construção de seus Sentidos: o ponto de vista dos profissionais de Saúde. Cad Saúde Publ 2002;18:168594.

3.Teixeira RR, Cyrino AP. As ciências sociais, a comunicação e a saúde Social. Ciênc Saúde Coletiva 2003;8:151-72. http://dx.doi.org/10.1590/S1413-81232003000100012

4.Ferreira VR, Carvalho LBC, Rutuolo F, Morais JF, Prado LBF, Prado GF. Sleep Disturbance Scale for Children: Translation, cultural adaptation and validation. Sleep Med 2008;10:457-63. http://dx.doi.org/10.1016/j.sleep.2008.03.018

5.Beebe DW. Neural and neurobehavioral dysfunction in children with obstructive sleep apnea. Plos Med 2006;3:1220-1. http://dx.doi.org/10.1371/journal.pmed.0030323

6.Carvalho LBC, Prado, LBF, Silva L, de Almeida MM, Almeida e Silva $\mathrm{T}$, Lora MI, et al. Cognitive Dysfunction in children with sleepdisordered breathing. J Child Neurol 2005;20:400-4. http://dx.doi.org/10.1177/08830738050200050101

7.Gozal D, O'Brien L, Row BW. Consequences of snoring and sleep disordered breathing in children. Ped Pulmonol 2004;26(suppl):1668.

8.SUCEN. Educação e Prevenção (endereço de internet). Acessado em: 24/11/2004. Disponível em:

http://www.sucen.sp.gov.br/educacao/index.htm

9.Schall VT, Juberg P, Rozemberg B, Vasconcellos MC. Ciranda da Saúde: Aprendendo e Ensinando através de Histórias infantis no 10 grau. Ciênc Cult 1986;38(suppl 7):159.

10.RoschkeMAC(ed). Evaluación en procesos de educación permanente y capacitación en salud: experiencias y lecciones. Washington DC: Organización Panamericana de la Salud, 2006, 206p. 11.Anuário Estatístico do Estado de São Paulo (endereço de internet). Acessado em: 11/11/2004. Disponível em: www.seade.gov.br/produtos/anuario/

12.IBGE - Instituto Brasileiro de Geografia e Estatísitca (endereço de internet). Acessado em: 20/01/2009. Disponível em: http://www.ibge.br 
13.Barros JC, Samara BS. Pesquisa de Marketing, conceitos e Metodologia. São Paulo: Person Education do Brasil, 2002, 220p.

14.Schall VT, Dias AP, Malaquias MLG, Santos MG. Educação em Saúde em Escolas Públicas de 10 grau da periferia de Belo Horizonte, MG, Brasil. I - Avaliação de um Programa Relativo à Esquistossomose. Rev Instituto Med São Paulo 1993;35:563-72. http://dx.doi.org/10.1590/S0036-46651993000600014

15.Kamel CR. Ciências e quadrinhos: explorando as potencialidades das histórias como materiais instrucionais (Dissertação). Rio de Janeiro: Instituto Oswaldo Cruz; 2006,129 p.

16.Camargo EP, Prado GF. Design da Informação: Desenvolvimento e Seleção de Personagens para Material Informativo, Aplicado À Educação Em Saúde Pediátrica. Blucher Design Proceedings 2014:1(4):67-78. http://dx.doi.org/10.5151/designpro-ped-00670

17.Santos MB, Vasconcellos SA, Dias RA, Oliveira LR, Ragozo AMA, Nori MTM, et al. Educação em saúde aplicada a prevenção de larva Migrans Visceral. Comparação da Eficiência de cinco recursos pedagógicos. Vet Zootec 2005;12:29-41.

18. Moya A. Shazam! 3a ed. São Paulo: Perspectiva, 1977, 343p.

19. Eisner W. Comics and Sequential Art, 1985. 154p.

20.Rota G, Izquierdo J. "Comics" as tool for teaching biotechnology in primary schools. Issues biotechnol Teach 2003;6:85-9.

21. Caruso F, Silveira C. Quadrinhos para a cidadania. Hist Cienc Saude Manguinhos 2009;16:217-236.

http://dx.doi.org/10.1590/S0104-59702009000100013

22. Roschke MAC (ed). Evaluación en procesos de educación permanente y capacitación en salud: experiencias y lecciones Washington DC: Organización Panamericana de la Salud, 2006, 206p. 23. Atkin CK, Rice RE. Public Communication Campaigns. 3a Ed. Newbury Park: SAGE Publications, 2000, 428p. http://dx.doi.org/10.4135/9781452233260

24.Oliveira KS. Avaliação do material didático do projeto "Criança saudável - educação dez", ano 2005. Interface - Comunic Saúde Educ 2008;12:401-10.

http://dx.doi.org/10.1590/S1414-

32832008000200014

25.Adam K. Dietary habit and sleep after bedtime food drinks. Sleep $1980 ; 3: 47-58$.

26. Lindsley IG, Hartmann E, Mitchell W. Selectivity in response to Itryptophan among insomniac subjects: a preliminary report. Sleep 1983;6:247-56. http://dx.doi.org/10.1093/sleep/6.3.247

27. Beiras A, Lodetti A, Cabral A, Grimm T, Maria JF, Raimundo P. Gênero e super-heróis: o traçado do corpo masculino pela norma. Psicol Soc 2007;19:62-7. http://dx.doi.org/10.1590/S010271822007000300010

28. Hicks RA, Bautista J, Mccullough M. Snoring may be linked to Culture. Medical Hypotesys 1994;42:24-6. http://dx.doi.org/10.1016/0306-9877(94)90030-2 
29. Camargo EP, Carvalho LBC, Prado LBF, Prado BF. Marketing Social: Caracterização do Paciente do Ambulatório de Neuro-sono da UNIFESP. Rev Neurocienc 2011;19:200-9.

http://dx.doi.org/10.4181/RNC.2010.05ip.09

30.Camargo EP, Carvalho LBC, Prado LBF, Prado BF. Is the population properly informed about sleep disorders? Arq Neuropsiquiatr 2013;71(2):92-9. http://dx.doi.org/10.1590/S0004-

$\underline{282 \times 2013005000001}$ 